\title{
Cohabitation with farm animals in urban households with and without occupational farm work: associations between participation in educational activities and good hygiene practices in at-risk households cohabiting with farm animals
}

\author{
Phoupasong Somphou • Takehito Takano • \\ Keiko Nakamura
}

Received: 16 April 2008/Accepted: 22 July 2008/Published online: 21 August 2008

(c) The Japanese Society for Hygiene 2008

\begin{abstract}
Objectives This study was performed to investigate patterns of cohabitation with farm animals in urban households in Vientiane, Lao People's Democratic Republic, with regard to animal-to-human disease transmission. We also investigated the association between participation in hygiene-related educational activities and good hygiene practices in households with or without cohabitation with animals.

Methods A survey regarding cohabitation with animals, socioeconomic characteristics and participation in educational activities was conducted among 1,497 households randomly sampled from urban districts of Vientiane in 2001. Rates of satisfactory performance of recommended good hygiene practices according to a program commencing in 1996 were compared among households cohabiting with animals with or without participation in educational activities (reference group).

Results Even among households not engaged in agriculture as a major source of income, 54.4, 34.9, 7.9, 3.1 and $35.7 \%$ cohabited with chickens, ducks, cattle, buffaloes and dogs, respectively. The percentage of households
\end{abstract}

P. Somphou · T. Takano

Health Promotion Section, Division of Public Health,

Graduate School of Tokyo Medical and Dental University,

Tokyo, Japan

P. Somphou

Vientiane Municipality Health Department,

Vientiane, Lao People's Democratic Republic

K. Nakamura $(\square)$

International Health Section, Division of Public Health, Graduate School of Tokyo Medical and Dental University, Yushima 1-5-45, Bunkyo-ku, Tokyo 113-8519, Japan

e-mail: nakamura.ith@tmd.ac.jp fulfilling the recommendations for good hygiene practices was $56.7 \%$. The rates of satisfactory hygiene practices among households participating in health education and cohabitating with chickens, ducks or cattle were greater than those in the reference group $(\mathrm{OR}=1.7,95 \% \mathrm{CI}=1.2$, $2.3 ; \mathrm{OR}=2.0,95 \% \mathrm{CI}=1.3,3.0 ; \mathrm{OR}=2.3,95 \% \mathrm{CI}=1.0$, 4.9) regardless of socioeconomic factors. Households cohabiting with animals showed poorer rates of satisfactory hygiene practices than those without animals.

Conclusions Cohabitation with farm animals is common in urban Vientiane regardless of household involvement in agriculture. Further effort is required to improve hygiene conditions, despite some positive effects of health education even in households cohabiting with animals.

Keywords Farm animals - Household hygiene .

Evaluation · Village-based activities .

Health communication

\section{Introduction}

Farm animals reared in or around residences have been shown to be sources of animal-transmitted diseases [1, 2], and living in an urban area with a high density of cohabitation with animals is regarded as a risk factor of such infection. However, living closely with farm animals has long been part of people's lifestyle in various regions around the world and provides benefits with regard to nutrition for the family and contribution to the household finances [3]. Due to the global increases in urbanisation, international commerce and international information spread, people's lifestyles and residential hygiene conditions are changing over time. A lack of information regarding the patterns of cohabitation with animals in 
urban areas hampers the design of effective measures of disease prevention to protect the health or urban dwellers where many people live in close proximity with domestic animals.

The recent worldwide spread of avian influenza and reports of its human cases have highlighted the urgent need for prevention of infectious diseases transmitted by animals. A World Health Organization (WHO) report included laboratory test results that confirmed 379 human cases of avian influenza worldwide, including 239 deaths, by 8 April 2008 [4]. While greater total numbers of deaths were reported in Indonesia (107 deaths/132 cases) and Vietnam (52 deaths/106 cases), all of the confirmed cases in Cambodia ( 7 cases, all since 2005), Lao People's Democratic Republic (Lao PDR, 2 cases, all since February 2007) and Nigeria (1 case, in January 2007) ended in death of the patient [4]. The majority of human cases of avian influenza were not associated with working on large poultry farms, but rather were among people coming into close contact with poultry in their backyards or homes [5, 6]. Therefore, the risk of animal-to-human transmission of this disease in places close to human residences is a significant health concern.

There is concern regarding the potential for animal-tohuman transmission of avian influenza through water and sewage due to the presence and stability of avian influenza virus in such environments [7, 8]. With regard to the transmission of other infectious diseases, previous reports have indicated the persistence of pathogens in animal and human excreta and in waste water [9, 10]. Although the transmission pathways of avian influenza from poultry to humans have not been clearly identified in all cases reported to date, the WHO recommends improvement of hygiene to reduce the risks of transmission of avian influenza to humans and the potential transmission of pandemic H5N1 if and when such a virus emerges [11]. Therefore, "good hygiene practices", such as access to clean water, drinking safe water, washing hands with soap and water, maintaining a clean and organised household environment, separating materials used inside a residence from those designed for outside use to avoid contamination and wellmaintained drainage of sewage are encouraged to reduce risk of animal-to-human infections as well as to reduce the risks of other infectious diseases.

Lao PDR is a landlocked mountainous country in Southeast Asia, which shares borders with China's Yunnan Province, Myanmar, Thailand, Cambodia and Vietnam. The National Household Survey of a total of 867,000 households nationwide performed in 2003 indicated that $28 \%$ were in urban areas [12]. Lao society is highly dependent on agriculture, and even among households in urban areas, farm work is the main economic activity of $34 \%$ of all urban households and of $31 \%$ of all households in the capital, Vientiane [12]. Agriculture constitutes a significant part of the urban lifestyle even in the most urbanised areas in the country.

The Ministry of Health of Lao PDR has promoted health education since 1983 under the "Three-Clean" campaign, representing "eat clean, drink clean and stay clean", in accordance with an emphasis on hygiene in disease prevention. In addition to the general encouragement of a hygienic lifestyle by the national authorities, Vientiane Municipality has run a community-wide "Three-Clean" program at the village level since 1996 [13]. This activity was promoted under the Healthy Cities Vientiane Project, which encouraged people to commit themselves to making their own villages clean and healthy places to live [14].

Household-level inspections had been carried out at least once for each of the houses in the villages of urban districts in Vientiane between 1996 and 2001 to identify the levels of cleanliness in individual households and then to identify the levels of household hygiene of each village. In accordance with the Healthy Village Program guidelines, meetings with public health professionals regarding good hygienic practices, meetings organised by village health volunteers to promote good hygiene practices and household visits by village health volunteers to provide advice regarding good hygiene practices have been carried out. The time and place of the meetings are determined in accordance with the preferences of the village heads and villagers.

In addition to village-based hygiene-related educational activities, the municipality used various media and promotional materials to disseminate information on good hygiene practices. Households vary in their participation in education activities and access to health information through media and promotional materials. Therefore, insufficient participation of some households in educational activities and lack of access to health-related information are caused for concern from the viewpoint of public health. Furthermore, due to the increased health risks of households cohabiting with animals, there is particular concern among public health professionals regarding the associations between such educational activities and information regarding good hygiene practices in households cohabiting with animals in Vientiane, Lao PDR.

This study was performed to investigate the patterns of cohabitation with farm animals in urban households in Vientiane, Lao PDR, with reference to concerns about animal-to-human transmission of diseases. Furthermore, the associations between participation in hygiene-related educational activities, information seeking in promotion of hygiene and satisfaction of recommended good hygienic practices by households with or without animal cohabiting were investigated. 


\section{Methods}

Study area and subjects

The study was performed in two districts selected at random from among five urban districts in Vientiane Municipality, Lao PDR. The area was located within a radius of $35 \mathrm{~km}$ from the capital city's central intersection and housed 120,459 residents. Seventeen of the 98 villages in this area were selected at random by drawing lots, and one-third of the households identified in each village office were selected for this study. The subject households were selected by taking every third household in the village registration records. If no member of a household could be contacted within three consecutive days (including visits in the evening and on weekends), another household not selected by earlier visits and located near by was contacted for participation in the study. A total of 1,497 households completed the household survey and inspection, which were carried out between February and March 2001. Among them, 1,472 households (98.3\%) were on the initial list and 25 households were replacements for others on the initial list.

\section{Procedures}

A household interview survey of individual households was conducted by door-to-door visits by village health workers familiar with the everyday lifestyle of the village households. Structured questionnaire forms, prepared in the Lao language, were pre-tested in the field to ensure that all questions could be understood clearly. The interview team members participated in a 3-day training workshop prior to the survey to standardise the interviews, hygiene inspections and data recording through practice visits to households in communities.

The ethical appropriateness of the survey procedures was approved by both the National Healthy City Team of Lao PDR and the Vientiane Municipal Government. Before the village households were visited, the purpose of the survey and its procedures were explained to the administrators of relevant sectors of the city government, including the health department, the governors of the districts involved, all of the village leaders of the villages included in the survey, the volunteer interviewers and the National Healthy City Team from the national government's Ministry of Health. Each interview was conducted only after obtaining the interviewee's informed consent to participate in the survey. Before administering the questionnaire and inspecting the household's dwelling and land, the interviewers explained the purpose of the study and informed consent was obtained from all respondents. The respondents of the survey consisted of 849 and 397 principle male and female adult household members, respectively, and 251 other relatives over 18 years old.

\section{Assessment variables}

The following items were assessed by the interviews and inspections.

\section{Cohabitation with farm animals}

With regard to cohabitation with farm animals, the numbers of chickens, ducks, cattle and buffaloes reared within each household's lot were examined. With regard to nonfarm animals, the number of dogs reared within each household's lot was also examined. The household's lot included the house building itself and sheds or land for animals adjacent to the house. The sums of the numbers of animals reared and present in and around the household's lot during the day or night-time were counted. Households with at least one of each type of farm animal were regarded as cohabitating with that animal.

\section{Socioeconomic characteristics and farm land}

The occupation(s) of the household members and education history of the principal male and female adult members of the households, as well as the total income of all adult household members were examined. The occupation associated with the main source of household income was classified as "agriculture farm work", "government work", "workers, including factory workers, manufacturing, labourers and merchants" or "others". In further analyses, work other than agriculture farm work was classified as "non-agriculture farm work". We refer to "agriculture households" and "non-agricultural households" as those for which agricultural work or nonagricultural work was the main occupation, respectively. Educational attainments of the principal male and female adult household members, who were defined as married couples and were normally younger than 50 and lived with or without their children, were determined. If there were two generation couples with their respective children living in the same household, the younger couple were defined as the principle male and female members of these families. Completion of at least high school was regarded as "higher educational status" for both male and female household members. Monthly household income was recorded. Households with monthly income of 1,000,000 kip (equivalent to approx. \$100 US) or more were regarded as "higher income households", according to the share of the upper $11.4 \%$ of the households. Possession of at least some farm land, except a small backyard vegetable garden less 
than $10 \mathrm{~m}^{2}$, was regarded as "owning some farm land", while the rest were regarded as "not owning farm land."

\section{Household hygiene}

The following four variables were assessed by inspection: (1) access to piped water; (2) a clean, organised yard with separation from farm animals; (3) well-maintained drainage and (4) tidiness inside the house with separation of materials designated for inside and outside use. Two variables with regard to hygiene-related practices were also defined according to the reports by the respondents: (5) family's practice of boiling water before drinking; and (6) family's practice of washing hands before eating. Each variable was scored as either "yes" or "no". After calculating a sum of scores of all six variables ranging from 0 to 6 , households with scores of five or six were defined as households performing the recommended good hygiene practices. These criteria were given as recommendations according to the village activities since 1996, and were used for evaluation of household hygiene-related conditions in their community activities.

\section{Participation in hygiene-related educational activities and sources of hygiene information}

The frequency of participation in village-based hygienerelated educational activities in the previous 12 months intended to prevent infectious diseases was recorded as "none", "sometimes", "often" or "always". Households are called to attend hygiene-related educational activities five to six times per year in the districts studied. "Sometimes" was regarded as participation in one to two times/ year, while "often" was regarded as participation three to four times/year. Households that reported participation in hygiene-related educational activities as "always" or "often" were classified as "participation in hygiene-related educational activities at least three times in the previous year". Respondents were asked about the sources from which they and their families obtain health-related information from among the following: television, radio, newspapers and health-related information posters.

\section{Statistical analysis}

The patterns of cohabitation with farm animals in urban Vientiane were examined based on the following indicators: (1) percentages of cohabitation with farm animals by type by household occupation; (2) difference in distributions of cohabitation with farm animals among by household occupation by chi-square test and (3) percentages of households cohabiting with farm animals by types of animals and by ownership of farm land among agricultural and non-agricultural households.

Percentages of households satisfied the six individual criteria to evaluate performance of good hygiene and Spearman's correlation coefficients among the six variables were calculated.

The characteristics of households that performed recommended good hygiene practices were examined. The percentages of households performing good hygiene practices were calculated according to cohabitation with animals, agriculture farm work as the household's main occupation, ownership of farm land, education, income, participation in hygiene-related educational activities and sources of hygiene information.

Logistic regression analyses were performed to examine the associations between hygiene practices of the households and participation in hygiene-related educational activities and cohabitation with particular animals, after adjustment for the influence of agriculture farm work as the main household occupation, education and income variables. Households that did not participate in hygienerelated educational activities and had no cohabitation with animals were used as a reference category, and odds ratios for three other categories (not participating in hygienerelated educational activities and no cohabitation with animals; participating in hygiene-related educational activities and cohabitation with animals; and participating in hygiene-related educational activities and no cohabitation with animals) were calculated.

Similar analyses were performed to examine the associations between hygiene practices of the households and seeking health information from the radio or newspaper by the households and cohabitation with particular animals, after adjustment for the influence of agriculture farm work as the main household occupation, education and income variables. The choice of radio and newspaper as the assessment variable was determined according to the percentages of households performing "hygiene practices".

\section{Results}

Of the 1,497 subject households in Vientiane, 60.5 and $39.8 \%$ cohabitated with chickens and ducks, respectively, while 13.6 and $7.3 \%$ cohabitated with cattle and buffaloes, respectively. Of the households included in the study, $60.3 \%$ reported cohabitated with dogs. Table 1 shows the distribution of cohabitation with animals among households with and without agriculture farm work as the main occupation. The percentages of non-agricultural households cohabitating with chickens, ducks, cattle, buffaloes and dogs were 54.4, 34.9, 7.9, 3.1 and $35.7 \%$, respectively. 
Table 1 Cohabitation with animals by household occupation $(n=1,497)$

\begin{tabular}{|c|c|c|c|c|c|}
\hline & All $(n=1,497) \%$ & $\begin{array}{l}\text { Agriculture farm } \\
\text { work }(n=422) \%\end{array}$ & $\begin{array}{l}\text { Government workers } \\
(n=330) \%\end{array}$ & $\begin{array}{l}\text { Workers (factory works, } \\
\text { manufacturing, labourers } \\
\text { and merchants) }(n=338) \%\end{array}$ & Others $(n=222) \%$ \\
\hline \multicolumn{6}{|c|}{ Chickens } \\
\hline 0 & 39.5 & 25.8 & $43.3 * * *$ & $50.9 * * *$ & $42.3 * * *$ \\
\hline $1-9$ & 28.6 & 39.3 & 18.8 & 26.6 & 25.7 \\
\hline$>10$ & 31.9 & 34.8 & 37.8 & 22.5 & 32.0 \\
\hline \multicolumn{6}{|c|}{ Ducks } \\
\hline 0 & 60.2 & 49.8 & $59.4 *$ & $67.8 * * *$ & $69.8 * * *$ \\
\hline $1-9$ & 23.9 & 30.1 & 23.9 & 20.1 & 18.0 \\
\hline$>10$ & 15.9 & 20.1 & 16.7 & 12.1 & 12.2 \\
\hline \multicolumn{6}{|c|}{ Cattle } \\
\hline 0 & 86.4 & 72.0 & $93.0 * * *$ & $94.4 * * *$ & $91.4 * * *$ \\
\hline$>1$ & 13.6 & 28.0 & 7.0 & 5.6 & 8.6 \\
\hline \multicolumn{6}{|c|}{ Buffaloes } \\
\hline 0 & 92.7 & 82.7 & $97.0 * * *$ & $98.8 * * *$ & $95.9 * * *$ \\
\hline$>1$ & 7.3 & 17.3 & 3.0 & 1.2 & 4.1 \\
\hline \multicolumn{6}{|l|}{ Dogs } \\
\hline 0 & 59.7 & 49.8 & $65.5 * * *$ & $63.0 * * *$ & $64.4 * * *$ \\
\hline$>1$ & 40.3 & 50.3 & 34.5 & 37.0 & 35.6 \\
\hline
\end{tabular}

Chi-square test compared with distributions in households with agriculture farm work as the main occupation

$* P<0.05, * * * P<0.001$

The distributions significantly differed by type of animals and by household occupation.

The percentages of agricultural households cohabiting with chickens, cattle and buffaloes were similar regardless of the ownership of farm land. Ducks and dogs were more commonly kept by agricultural households owning some farm land (54.7 and 53.4\%, respectively) than those owning no such land (36.3 and 40.2\%, respectively). Among nonagricultural households, the percentages of cohabitation with chicken, ducks, cattle and buffaloes were significantly greater in households owning some farm land (62.5, 41.1, 11.4 and $4.4 \%$, respectively) than in those not owning no such land (46.2, 28.5, 4.3 and $1.7 \%$, respectively).

Spearman's correlation coefficients among six variables to evaluate performance of recommended good hygiene practices are presented in the Table 2. The following pairs of variables showed statistically significant associations with Spearman's Correlation Coefficient greater than 0.3: HP2 variable (a clean, organised yard with separation from farm animals) and HP3 (well-maintained drainage), HP2 and HP4 (tidiness inside the house with separation of materials designated for use inside and outside), and HP3 and HP4.

With regard to the household hygiene conditions, $56.7 \%$ of the households performed the recommended hygiene practices. Table 3 shows the percentage of households performing the recommended hygiene practices according to household characteristics. Performance of hygiene practices by households was less likely to be observed among those households cohabiting with chickens, ducks, cattle, buffaloes or dogs than those without these animals. Non-agricultural households, owning no farm land and high educational attainment of principal male or female family members were associated with higher percentages of households performing the recommended hygiene practices than the other factors examined. Male and female educational variables correlated each other closely (Spearman's correlation coefficient $=0.532$ ) and showed similar relationship with good hygiene practices variable, female education variable was used for further analysis as an indicator of educational level. Household income showed no associations with household performance of good hygiene practices. Participation in hygiene-related educational activities and use of television, radio and newspaper as sources of hygiene information were associated with household performance in hygiene practices. The variables of use of radio and newspaper showed strong significant associations with good hygiene practices. These two variables were used to refer health information seeking behaviour in the further analysis.

Table 4 shows the factors associated with performance of the recommended hygiene practices. Combined status of not participating in hygienic education and cohabiting with animals was used as a reference group. In 
Table 2 Correlation coefficients of variables to evaluate recommended good hygiene practices

\begin{tabular}{|c|c|c|c|c|c|c|c|c|}
\hline \multirow[t]{2}{*}{ Variable } & \multirow[t]{2}{*}{ Criteria } & \multirow{2}{*}{$\begin{array}{l}\% \text { Satisfying } \\
\text { individual criteria }\end{array}$} & \multicolumn{6}{|c|}{ Spearman's correlation coefficients } \\
\hline & & & HP1 & HP2 & HP3 & HP4 & HP5 & HP6 \\
\hline HP1 & Access to piped water & 52.7 & - & $0.11 * *$ & 0.03 & $0.12 * *$ & 0.01 & 0.01 \\
\hline HP2 & A clean, organised yard with separation from farm animals & 29.0 & & - & $0.33 * *$ & $0.41 * *$ & $0.09 * *$ & 0.03 \\
\hline HP3 & Well-maintained drainage & 89.9 & & & - & $0.46^{* *}$ & 0.03 & 0.03 \\
\hline HP4 & $\begin{array}{l}\text { Tidiness inside the house with separation of materials } \\
\text { designated for inside and outside use }\end{array}$ & 85.5 & & & & - & 0.00 & $0.05^{*}$ \\
\hline HP5 & Practice of boiling water before drinking & 33.5 & & & & & - & 0.05 \\
\hline HP6 & Practice of washing hands before eating & 98.4 & & & & & & - \\
\hline
\end{tabular}

$* P<0.05, * * P<0.01$

Table 3 Percentage of households performing recommended good hygiene practices by household characteristics

\begin{tabular}{|c|c|c|}
\hline & $N$ & $\begin{array}{l}\% \text { Households performing recommended } \\
\text { good hygiene practices }\end{array}$ \\
\hline Cohabiting with chicken(s), yes/no & $742 / 490$ & $51.2 / 64.9 * * *$ \\
\hline Cohabiting with duck(s), yes/no & $503 / 729$ & $50.1 / 61.2 * * *$ \\
\hline Cohabiting with cattle, yes/no & $178 / 1,054$ & $40.4 / 59.4 * * *$ \\
\hline Cohabiting with buffalo(es), yes/no & $85 / 1,147$ & $32.9 / 58.4 * * *$ \\
\hline Cohabiting with $\operatorname{dog}(\mathrm{s})$, yes/no & $507 / 725$ & $53.1 / 59.2^{*}$ \\
\hline Agricultural farm work as the main occupation, yes/no & $355 / 877$ & $36.1 / 65.0 * * *$ \\
\hline Farm land, some/none & $759 / 473$ & $49.7 / 67.9 * * *$ \\
\hline Education, male principal, high school or higher/other & $408 / 824$ & $66.4 / 51.8 * * *$ \\
\hline Education, female principal, high school or higher/other & $186 / 1,046$ & $71.0 / 54.1 * * *$ \\
\hline $\begin{array}{l}\text { Household income, high }(\geq 1 \text { million kip/month }) / \\
\text { low }(<1 \text { million kip/month) }\end{array}$ & $139 / 1,081$ & $61.9 / 55.9^{\mathrm{ns}}$ \\
\hline $\begin{array}{l}\text { Participation in hygiene-related educational activities, } \\
\text { at least three times in the previous year, yes/no }\end{array}$ & $830 / 402$ & $59.4 / 51.0 * *$ \\
\hline \multicolumn{3}{|l|}{$\begin{array}{l}\text { Sources from which household members seek } \\
\text { hygiene-related information }\end{array}$} \\
\hline Television, yes/no & $763 / 469$ & $59.8 / 51.6^{* *}$ \\
\hline Radio, yes/no & $226 / 1,006$ & $71.7 / 53.3 * * *$ \\
\hline Newspaper, yes/no & $96 / 1,136$ & $80.2 / 54.7 * * *$ \\
\hline Health-related posters, yes/no & $106 / 1,126$ & $48.1 / 57.5^{\mathrm{ns}}$ \\
\hline
\end{tabular}

Recommended good hygiene practices were defined as satisfying five or more of the following six criteria: (1) access to piped water; (2) a clean, organised yard with separation from farm animals; (3) well-maintained drainage; (4) tidiness inside the house with separation of materials designated for inside and outside use; (5) family's practice of boiling water before drinking and (6) family's practice of washing hands before eating. Criteria (1)-(4) were evaluated by inspection, and (5) and (6) were evaluated by self-reporting of household members

1 million kip equivalent to $\sim 100$ USD

$n s$ not significant

$* P<0.05, * * P<0.01, * * * P<0.001$

comparison with the reference group, households participating in hygiene-related educational activities and cohabitating with animals were more likely to perform the recommended hygiene practices, regardless of socioeconomic conditions, except in the case of those households keeping buffaloes. Among the households participating in hygiene-related educational activities, the odds ratios for performance of the recommended hygiene practices were greater in the households without than in those living with animals.

Table 5 shows the factors associated with performance of the recommended hygiene practices by combined the 


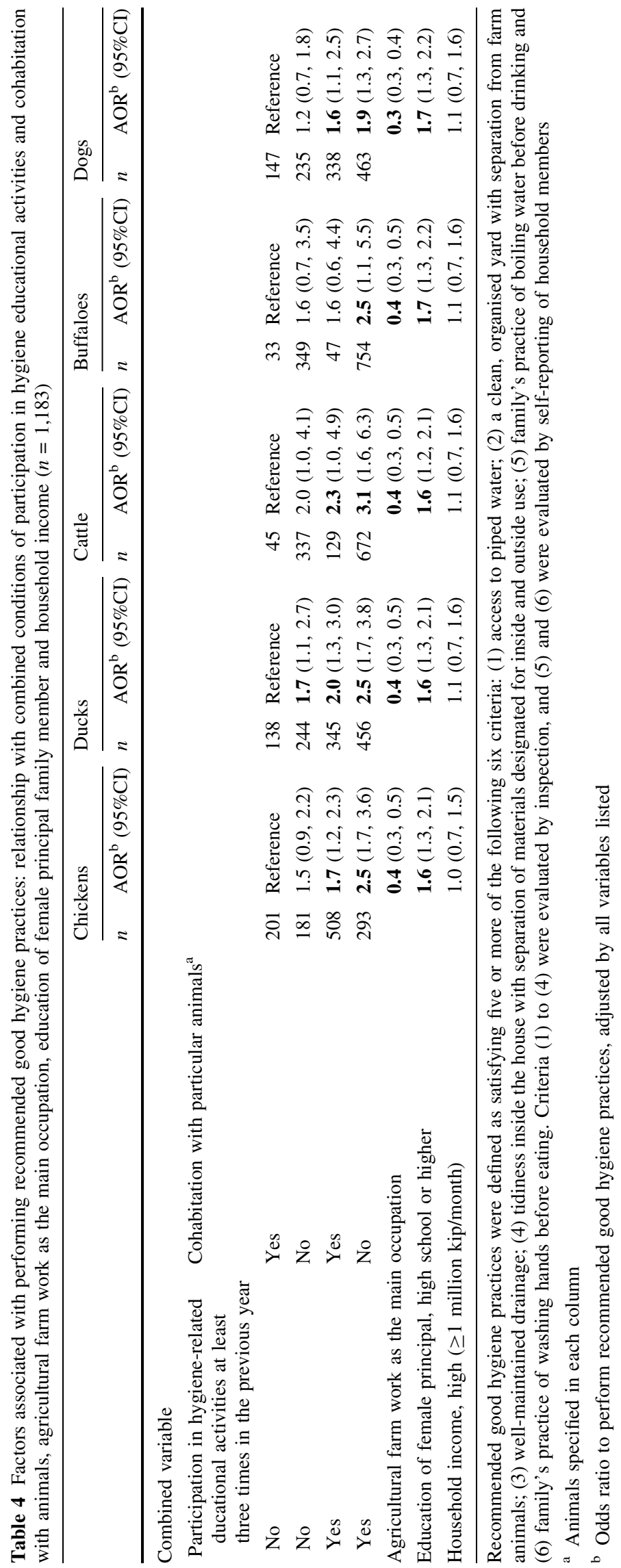


variables of seeking health information and cohabitation with animals. A combined status of not seeking health information from the radio or newspaper and cohabiting with animals was used as a reference group. In comparison with the reference group, households seeking health information from the radio or newspaper and cohabiting with chickens were more likely to be associated with performance of the recommended hygiene practices, regardless of socioeconomic conditions. Among the households seeking health information from the radio or newspaper, the odds ratios for performance of the recommended hygiene practices were greater in the households without than in those with animals.

\section{Discussion}

The results of the present study revealed variations in household cohabitation with farm animals in urban areas in Vientiane. Not only agricultural households but also nonagricultural households in urban areas cohabitated with farm animals. The percentage of households performing good hygiene practices was smaller in agricultural households than in non-agricultural households, and was smaller in those households with than in those without farm animals. Participation in hygiene-related educational activities was associated with better performance in the recommended hygiene practices among those households cohabiting with animals. Statistically significant relationships were observed for households rearing chickens, ducks, cattle or dogs, regardless of socioeconomic conditions. Seeking health information from the radio or newspaper was associated with better performance of the recommended good hygiene practices among households cohabiting with chickens or dogs, regardless of socioeconomic conditions.

The agricultural census, which includes information from farm households in Lao PDR, indicated that 73, 31 and $48 \%$ of farming households kept chickens, cattle or buffaloes [15]. The percentages of farming households in urban Vientiane cohabiting with chickens and cattle were similar to those of animal holders among farming households throughout the country. Cohabitation with buffaloes was less frequent in urban Vientiane than for farming households with buffaloes throughout the country. Information on animal holdings in urban areas is rather limited. Considering the similar percentage of animal holding among farming households in urban Vientiane compared with that among farming households in the country as a whole, further studies should be performed to gauge the potential reservoir of animal-tohuman transmission of diseases in densely populated areas. 
The study population was a representative sample of urban Vientiane selected by random sampling. Information on animal rearing was obtained from both households for which agriculture was not the main source of income in urban districts. Although the percentages of cohabitation with animals among urban non-agricultural households were lower than those among urban agricultural households, it should be noted that a large proportion of households reported cohabiting with farm animals even among those households not engaged in agriculture. This observation suggested that there is a widespread risk of backyard infection by animal-transmitted diseases in populated urban centres in Lao PDR. The risk is spread not only among farming households, but also among nonfarming households, even among those without any farm land. Information on the number of at-risk households and on target households requiring more intensive intervention will enable strategic planning in disease prevention programs.

This study showed that half of non-agricultural households in Vientiane reared chickens. A previous report on domestic animals in urban areas indicated a higher percentage of keeping poultry among urban households [3]. Poultry are small animals and rearing of such animals requires relatively little space, which allows non-agricultural households to rear animals even in an urban environment [16]. In contrast, large animals are usually reared by agricultural households or by non-agricultural households with farm land. In the latter type, household members themselves are not necessarily directly involved in animal rearing. Animals are regarded as a source of wealth in Lao society and keeping livestock contributes to the household finances [17]. Further information on handling practices of animals including use of animals for their family's food or selling of animals at markets as well as rearing methods identify points of contacts with higher risk of infection when the animals reserve pathogens. Information of which family members have contact with animals will facilitate identification of vulnerable family members at higher risk of potential animal-to-human disease transmission.

With regard to the associations between good hygiene practices and both participation in hygiene-related educational activities and health seeking behaviours, the results of the present study indicated better conditions among households without as compared to those with animals. Considering the results of the correlation analysis of individual variables to measure good hygiene, showing significant greater correlations among variables based on inspection and smaller correlation coefficients with variables based on self-report of the subjects, the selected six variables may represent more than one component of good hygiene practices. To further refer relationship between good hygiene and other factors, the components of good hygiene practices should be examined. In Vientiane, Lao PDR, chickens are generally housed in confined quarters at high density and are not perfectly isolated from wildfowl. Therefore, they are at high risk of infectious diseases spread by wildfowl [18]. The chickens reared by households with relatively large farm land areas represent different risks as they are kept free-range in people's backyards and are always exposed to potentially viruscontaminated materials [19]. Taking into consideration the lifestyle factors related to animal rearing in urban areas, dissemination of information on safe animal rearing practices with regard to prevention of the spread of infectious diseases, feeding with safe foods and complete isolation from wildfowl is recommended in communities where many households cohabit with animals.

The importance of strengthening the public health infrastructure and measures to prevent the spread of emerging and re-emerging infectious diseases has been emphasised repeatedly [20, 21]. Knowledge regarding the relative difficulties of achieving safe rearing of animals will facilitate promotion of a hygienic lifestyle as a basic measure to reduce the risk of spread of diseases. Specific information and preventive measures would work on the basis of promoting general behaviours associated with a hygienic lifestyle. Promotion of hygiene practices taking into consideration the lifestyles of households related to their occupation will further facilitate preparedness against animal-to-human disease transmission.

Cohabitation with farm animals is common in urban Vientiane regardless of whether the household is involved in occupational farm work. The association between village-based hygiene-related educational activities in urban districts and seeking hygiene information to promote good hygiene practices, required for prevention of animal transmitted diseases, was demonstrated in communities where urban agriculture is commonly practiced. Due to the increased risk of animal-to-human disease transmission among households cohabiting with animals with relatively unsatisfactory hygiene practices, further attention to improve hygiene conditions among these urban households cohabiting with animals is required, despite associations of health education even in households cohabiting with animals.

Acknowledgments This research was conducted as part of the Joint Research Project organised by Tokyo Medical and Dental University and Lao PDR Healthy Cities Research Team. The research was partly supported by Grants-in-Aide for Scientific Studies of Japan Society for the Promotion Science. The authors thank the project members, particularly Drs Bounlay Phommasack, Tayphasavanh Fengthong and Katthanaphone Phandouangsy (Department of Hygiene and Preventive Medicine, Ministry of Health, Lao PDR), Dr Somchit Akkhavong (Ministry of Health, Lao PDR) and Ratthyphone Oula (Vientiane Health Department, Lao PDR). 


\section{References}

1. Sere C, Neidhardt R. Urban-rural integration in peri-urban animal production. Agric Rural Dev. 1995;2:19-23.

2. Montovani A. Veterinary urban hygiene in developing countries. Urban Agric Mag. 2000;1:32-3.

3. Thys E, Oueadraogo M, Speybroeck N, Geerts S. Socio-economic determinants of urban household livestock keeping in semi-arid Western Africa. J Arid Environ. 2005;63:475-96.

4. World Health Organization. Cumulative number of confirmed human cases of avian influenza $\mathrm{A} /(\mathrm{H} 5 \mathrm{~N} 1)$ reported to WHO. Epidemiological Report of Cases of Avian Influenza. Geneva: World Health Organization; 2008.

5. Center for Disease Control and Prevention (CDC). Case of influenza A (H5N1)—Thailand. JAMA. 2004;53:100-3.

6. Tran TH, Nguyen TL, Nguyen TD, Luong TS, Pham PM, Nguyen $\mathrm{VV}$, et al. Avian influenza (H5N1) in 10 patients in Vietnam. $\mathrm{N}$ Engl J Med. 2004;350:1179-88.

7. Stallknecht DE, Shane SM, Kearney Mt, et al. Persistence of avian influenza viruses in water. Avian Dis. 1990;34:406-11.

8. Water, Sanitation and Health, Public Health and Environment, World Health Organization. Review of latest available evidence on potential transmission of avian influenza (H5N1) through water and sewage and ways to reduce the risks to human health. Geneva: World Health Organization; 2007.

9. Poon LLM, Guan Y, Micholls JM, Yuen KY, Peiris JSM. The aetiology, origins, and diagnosis of severe acute respiratory syndrome. Lancet Infect Dis. 2004;4:663-71.

10. Ensink JHJ, van der Hoek W, Mukhtar M, Tahir Z, Amerasinghe FP. High risk of hookworm infection among wastewater farmers in Pakistan. Trans R Soc Trop Med Hyg. 2005;99:809-18.
11. World Health Organization Department of Public Health and Environment and WASH Inter Agency Group. Questions and Answers on Potential Transmission of Avian Influenza (H5N1) through water, sanitation and hygiene and ways to reduce the risks to human health. Geneva: World Health Organization; 2007.

12. National Statistical Centre, Committee for Planning and Cooperation, Lao PDR. The household of Lao PDR: social and economic indicators Lao expenditure and consumption survey 2002/03 LECS3. Lao PDR: National Statistical Centre, Committee for Planning and Cooperation; 2004.

13. Vientiane Municipality. Promotion of sanitary practices by Healthy Village Programs. Vientiane Municipality, 2000.

14. Phommasack B. Lessons learned from the implementation of Healthy Cities in Lao People's Democratic Republic. In: Takano T, editor. Healthy cities and urban policy research. London: Spon Press; 2003. p. 225-33.

15. National Statistical Centre, Lao PDR. Agricultural Census, Lao PDR 1998/99. Lao PDR: National Statistical Centre; 2000.

16. Mougeot LJA. Urban agriculture: concept and definition. Urban Agric Mag. 2000;1:5-7.

17. Wilson RT. Status and prospects for livestock production in the Lao People's Democratic Republic. Trop Anim Health Prod. 2007;39:443-52.

18. Leslie D. Avian influenza in wild birds. Madison: National Wildlife Health Center (NWHC); 2000.

19. Horimoto T, Kawaoka Y. Pandemic threat posed by avian influenza A viruses. Clin Microbiol Rev. 2001;14:129-49.

20. Morse SS. Factors in emergence of infectious diseases. Emerg Infect Dis. 1995;1:7-15.

21. Louria DB. Emerging and re-emerging infections: the societal determinants. Futures. 2000;32:581-94. 